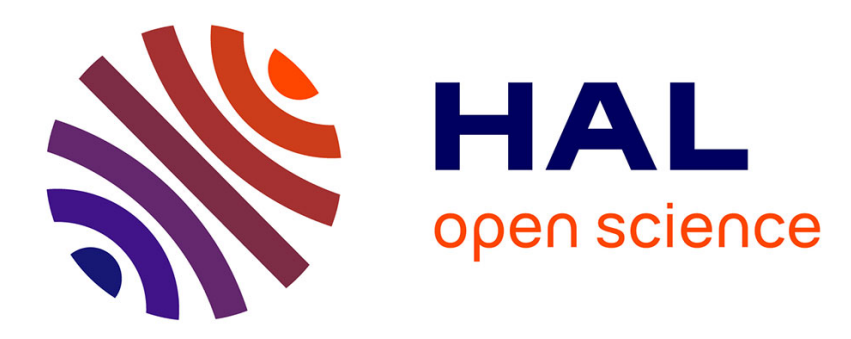

\title{
Statistical properties of an equation describing fluid interfaces
}

\author{
A. Pumir
}

\section{To cite this version:}

A. Pumir. Statistical properties of an equation describing fluid interfaces. Journal de Physique, 1985, 46 (4), pp.511-522. 10.1051/jphys:01985004604051100 . jpa-00209991

\section{HAL Id: jpa-00209991 https://hal.science/jpa-00209991}

Submitted on 1 Jan 1985

HAL is a multi-disciplinary open access archive for the deposit and dissemination of scientific research documents, whether they are published or not. The documents may come from teaching and research institutions in France or abroad, or from public or private research centers.
L'archive ouverte pluridisciplinaire HAL, est destinée au dépôt et à la diffusion de documents scientifiques de niveau recherche, publiés ou non, émanant des établissements d'enseignement et de recherche français ou étrangers, des laboratoires publics ou privés. 
Classification

Physics Abstracts

$47.10-47.25 \mathrm{C}$

\title{
Statistical properties of an equation describing fluid interfaces
}

\author{
A. Pumir (*) \\ Laboratory of Atomic and Solid State Physics, Cornell University, Ithaca, N.Y. 14853, U.S.A.
}

(Reçu le 24 septembre 1984, révisé le 5 décembre, accepté le 20 décembre 1984)

\begin{abstract}
Résumé. - On sait que les solutions de l'équation de Kuramoto-Sivashinsky, qui décrit des interfaces entre milieux fluides présentent du chaos, aussi bien temporel que spatial. Plusieurs propriétés statistiques de ce modèle ont été étudiées numériquement. On montre que les fluctuations d'une quantité locale ont une distribution fortement non gaussienne; l'influence des conditions aux limites et les phénomènes d'intermittence sont examinés. Les moments des fluctuations de la transformée de Fourier spatiale, naturellement reliés aux fonctions de corrélation ont été aussi étudiés. Aux grands nombres d'onde, les moments des fluctuations croissent plus vite que pour une distribution gaussienne, alors qu'aux bas nombres d'onde, les fluctuations sont pratiquement gaussiennes. Des effets de taille finie sont également discutés. De même, les fluctuations de la transformée de Fourier (temporelle) d'une quantité locale ont été étudiées, elles possèdent des propriétés analogues. Un comportement semblable est obtenu dans le cas des équations de Lorenz [23]. Enfin, on introduit des fonctions de corrélation testant la symétrie dans le renversement du temps, et on calcule plusieurs de ces fonctions. A l'aide des résultats numériques, on discute le processus de transfert d'énergie.
\end{abstract}

\begin{abstract}
The Kuramoto-Sivashinsky equation which describes fluid interfaces in several physical contexts is known to have chaotic solutions, displaying both space and time disorder. We have investigated numerically several statistical properties of this model. The fluctuations of a local quantity are shown to have a highly non Gaussian distribution; boundary effects and small scale intermittency phenomena are examined. The moments of the fluctuations of the space Fourier transform, which are related to the space correlation functions, are also investigated. The high order moments of large wavenumber fluctuations grow faster than the moments of a Gaussian variable; while the low wavenumber fluctuations are found to be almost Gaussian. Some finite size effects are also discussed. Similarly the fluctuations of the time Fourier transform of a local quantity have been investigated; they share most of the above mentioned property. A similar behaviour is observed in the case of the Lorenz [23] equations. Eventually we introduce correlation functions testing the time symmetry of the fluctuations and compute some of these functions. We use our numerical results to discuss energy transfer process.
\end{abstract}

\section{Introduction.}

In the past numerical simulations on simple model equations allowed a real improvement in our understanding of non linear dynamics. However many problems remain open. In the case of fully developed turbulence numerical studies are still limited (see, e.g. [1]). Experimentally the investigation of the statistical properties of turbulence is also a difficult task, though some progress has recently been accomplished (see e.g. [2]). From a theoretical point of view it remains difficult to get statistical information from the fluid dynamics equations; reliable results in this field are rather scarce. The importance of statistical properties

(*) Permanent address : Service de Physique Théorique, CEN Saclay, F-91191 Gif sur Yvette, France. in turbulent fluid systems is obvious since they are closely related to the quantities experimentally measured or relevant in practical applications. In order to get some information on a system displaying both spatial and temporal chaos we have investigated the Kuramoto-Sivashinsky equation (K.S.E.)

$$
\frac{\partial \phi}{\partial t}+\frac{\partial^{2} \phi}{\partial x^{2}}+\frac{\partial^{4} \phi}{\partial x^{4}}+\phi \frac{\partial \phi}{\partial x}=0
$$

$$
\begin{aligned}
x \in[0, L] & + \text { initial conditions } \\
& + \text { boundary conditions } .
\end{aligned}
$$

This equation has been derived by Kuramoto [3] to describe the evolution of a chemical system. It also arises in several physical contexts, involving fluid motions. In particular it has been derived by Sivas- 
hinsky to model the interface of a premixed flame front $[4,5]$ or the interface of a thin liquid film falling down an inclined plane [6,7]. In this study, the boundary conditions we have chosen were either $\phi=\partial \phi / \partial x=0$ at $x=0, L$, or periodic boundary conditions (the importance of these boundary conditions will be discussed below). The control parameter for the K.S.E. is the size $L$ of the system. As soon as $L$ is large enough, say $L \gtrsim 30$ a chaotic regime involving space and time disorder occurs. As one is concerned with a turbulent system, it makes sense to study statistical properties of the system. In particular, the spectrum of spatial fluctuations [8], and temporal fluctuations [9] are already known. Despite several theoretical attempts $[10,11]$ most of the noticeable features of these spectra remain ill understood. The K.S.E. have also been investigated from a dynamical systems standpoint $[12,13]$. Precise results concerning the Lyapunov exponents which measure the instability of trajectories in phase space [14] allow us to determine the amount of chaos, the number of turbulent degrees of freedom in the system, ... Obviously it would be worth connecting the approach in terms of dynamical systems, and the statistical description. In this work we have investigated numerically several statistical properties of the turbulent fluctuations, and tried to get information on dynamical processes involved by the K.S.E.

Thanks to efficient numerical algorithms (finite difference or pseudo-spectral, according to the nature of the boundary conditions) we could get statistical accurate results. The averages we have considered are time averages on one given trajectory. The present study suggests that our system has "good " ergodic properties, and that our statistical results characterize the system, and not a particular trajectory. Although this property might seem quite reasonable, it is not obvious at all if one thinks of low dimensional systems case [15].

In hydrodynamic turbulence experimentalists measure the fluctuations of local quantities such as one component of the velocity field, or its derivatives. In particular, the dimensionless ratios :

$$
\frac{\left\langle\left(\frac{\partial u_{i}}{\partial x_{i}}\right)^{4}\right\rangle}{\left\langle\left(\frac{\partial u_{i}}{\partial x_{i}}\right)^{2}\right\rangle} \text { (the flatness) or } \frac{\left\langle\left(\frac{\partial u_{i}}{\partial x_{i}}\right)^{3}\right\rangle}{\left\langle\left(\frac{\partial u_{i}}{\partial x_{i}}\right)^{2}\right\rangle^{3 / 2}}
$$

(the skewness)

provide some information on the probability distribution of the field $u$ (see [16]). In part. 2 we study similar quantities for the K.S.E. The correlation functions also provide much information on the system. For example since the work of Taylor, it is known that the 2 points correlation function is related to turbulent diffusion [17]. In part 3 we have investigated the nature of the fluctuations of the quantity $\phi(k)$, the spatial Fourier transform of $\phi$. These fluctuations depend on the size $L$ of the system we consider. The factorization property of the correlation functions, and some small scale intermittency effects are discussed. In the same spirit results concerning $n$ points time correlation functions are presented in part 4. A comparison with the Lorenz model is also studied. In order to investigate the dynamical processes in the system we have computed some correlation functions testing the time reversal symmetry of the fluctuations [18]. The results are described in part 5 . Eventually we present a few concluding remarks in part 6.

\section{One point statistics.}

In this paragraph we are dealing with the fluctuations of $\phi(x, t)$ and the flatness, skewness introduced in hydrodynamic turbulence (without the overall index complications, due to the simplicity of the 1-dimensional K.S.E.). In this study the boundary conditions were $\phi=\partial \phi / \partial x=0$ at $x=0, L$. Our time step was $\delta t=0.1$ and lattice spacing $\delta x=0.25$. These values were chosen so that $\sigma_{\max } \delta t \ll 1$ and $k_{\max } \delta x \ll 1$, where $k_{\max }$ is the linearly most unstable mode and $\sigma_{\max }$ the rate of growth of the linearly most unstable mode. The evolution of the solution, during a lapse of time $t=30$ is represented in figure 1 . The solution consists of bumps filling the whole domain in perpetual interaction. We have investigated the space dependence of the statistical properties in the system. As equation (1) is invariant under $x \rightarrow L-x$ and $\phi \rightarrow-\phi$ we restricted ourselves to the interval $0<x<L / 2(L=64$ or 128 in this study). Our numerical results were obtained from a sample of 21,600 values of the solution separated by $\Delta t=0.8$, which allows a good convergence of the statistical results.

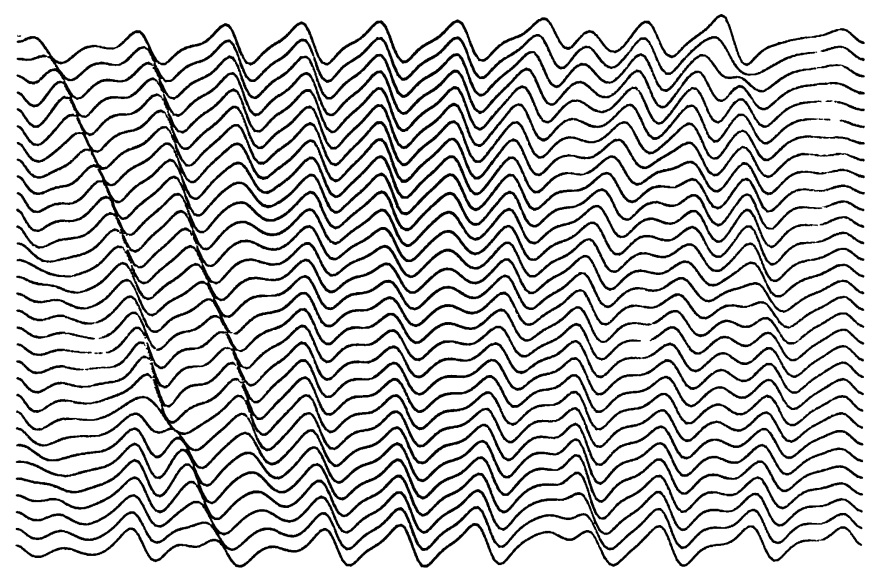

Fig. 1. - The evolution of the solution of the KuramotoSivashinsky equation during a time interval $\Delta T=30$. Two successive stages of the evolution are separated by $\Delta t=1.0$. The solution consists of bumps, filling the whole space, in perpetual interaction. 
Figure 2 displays the value of the $i$ th derivative flatness for $i=0,1,2,3,4$

$$
F i(x)=\frac{\left\langle\phi^{(i)}(x)^{4}\right\rangle}{\left\langle\phi^{(i)}(x)^{2}\right\rangle^{2}} ; \quad \phi^{(i)}=\frac{\partial^{i} \phi}{\partial x^{i}}
$$

as a function of $x$, while figure 3 displays the values of the $i$ th derivative skewness :

$$
\operatorname{Si}(x)=\frac{\left\langle\phi^{(i)}(x)^{3}\right\rangle}{\left\langle\phi^{(i)}(x)^{2}\right\rangle^{3 / 2}} .
$$

Two kinds of regime have been observed. First, near the boundaries the variable $\phi$ displays large fluctuations, which can be interpreted as follows. The strong constraints on the boundary

$$
\left(\left.\phi\right|_{\partial}=\left.\frac{\partial \phi}{\partial n}\right|_{\partial}=0\right)
$$

inhibits the occurrence of turbulent fluctuations. Consequently they are quite scarce and their distribution is very different, more intermittent than in the

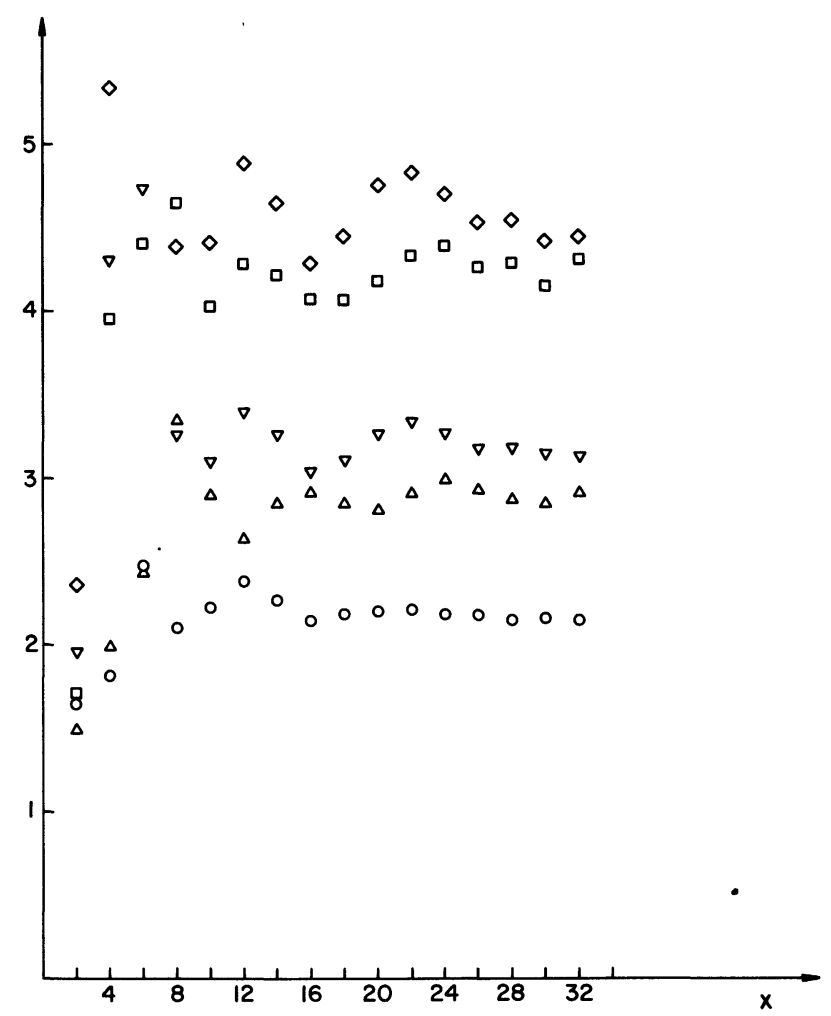

Fig. 2. - The dimensionless ratio $\left\langle\psi^{4}\right\rangle\left\langle\left\langle\psi^{2}\right\rangle^{2}\right.$ for several spatial derivatives of

$$
\phi\left(0, \phi ; \Delta, \frac{\partial \phi}{\partial x} ; \nabla, \frac{\partial^{1} \phi}{\partial x^{2}} ; \square, \frac{\partial^{3} \phi}{\partial x^{3}} ; \diamond, \frac{\partial^{4} \phi}{\partial x^{4}}\right),
$$

as a function of the position. The size of the system is $L=64$. The presence of the boundaries induces very intermittent fluctuations near the edges, while in the bulk an almost constant value is reached.

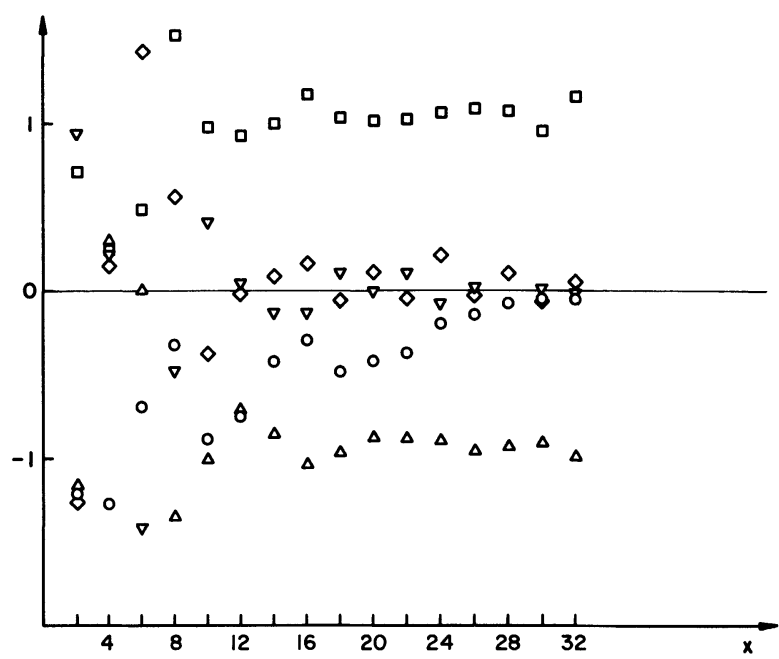

Fig. 3. - The dimensionless ratio $\left\langle\psi^{3}\right\rangle\left\langle\left\langle\psi^{2}\right\rangle^{3 / 2}\right.$ for several spatial derivatives of

$$
\phi\left(0, \phi ; \Delta, \frac{\partial \phi}{\partial x} ; \nabla, \frac{\partial^{1} \phi}{\partial x^{2}} ; \square, \frac{\partial^{3} \phi}{\partial x^{3}} ; \diamond, \frac{\partial^{4} \phi}{\partial x^{4}}\right)
$$

as a function of the position. The size of the system is $L=64$. The effects of the boundary are limited to a layer of size $L \sim 10$. The distribution of odd order derivatives is very asymmetrical.

bulk, as explained below. Obviously this would not occur with periodic boundary conditions. Similar phenomena are also observed in hydrodynamic turbulence. Secondly in the bulk the system forgets, in some sense the constraints of the boundary and the $F i$ reach a nearly constant value. Similar behaviours have also been observed for other ratios such as $\frac{\left\langle\phi^{(i)}(x)^{2 p}\right\rangle}{\left\langle\phi^{(i)}(x)^{2}\right\rangle^{p}}$ for $p>2$. The general shape of these functions is strongly reminiscent of the correlation functions in liquids. Far away from the boundary each of these dimensionless quantities reaches a nearly constant value with small damped oscillations, as for the density correlation functions in simple liquids. When $L$ is increased the size of the boundary layer remains almost constant. This size is of the order of magnitude of the size of the bumps in the solution (see Fig. 1). This length scale also arises from a linear stability analysis around the solution $\phi=0$ at the most unstable wavelength in the system.

From figure 3, it can be deduced that the distribution of odd-order derivatives of $\phi$ are very dissymmetrical. This phenomenon can be simply interpreted. The term $\left(\frac{\partial \phi}{\partial t}+\phi \frac{\partial \phi}{\partial x}\right)$ in the K.S.E. induces the formation of shocks. On figure 1 the shape of the bumps is reminiscent of these shocks. The lack of symmetry of such structures (one side of the bumps is steeper than the other) is responsible for the dissymmetrical nature of the odd order derivatives of $\phi$. Let us also mention that this dissymmetry has a simple physical 
origin in the case of a thin liquid film flowing down an inclined plane (in this case the variable $\phi$ describes the height of the film). The curves reported in figure 2 suggest that the dimensionless ratios

$$
\left\langle\left(\frac{\partial^{i} \phi}{\partial x^{i}}\right)^{4}\right\rangle /\left\langle\left(\frac{\partial^{i} \phi}{\partial x^{i}}\right)^{2}\right\rangle^{2}
$$

tend to increase with $i$, the order of the derivative. In the bulk we have tried to get more details on the statistical nature of the fluctuations. In figure 4 are presented for several values of $i$ (the order of the derivative) the logarithms of the dimensionless ratios $\frac{\left\langle\phi^{(i)}(L / 2)^{2 p}\right\rangle}{\left\langle\phi^{(i)}(L / 2)^{2}\right\rangle^{p}}$ as a function of $p(p=2,3, \ldots)$.

For a given value of $p$ the moments $\frac{\left\langle\phi^{(i) 2 p}\right\rangle}{\left\langle\phi^{(i) 2}\right\rangle^{p}}$ increase with $i$. This effect is similar to the effects of intermittency as discussed by Batchelor and Townsend [19]. One of the most important manifestation of the phenomenon of intermittency is the non Gaussian character of the fluctuations in the system. If the fluctuating real quantities $\psi=\frac{\partial^{(i)} \phi}{\partial x^{(i)}}$ were described by a centred Gaussian process one would obtain

$$
\frac{\left\langle\psi^{2 p+1}\right\rangle}{\left\langle\psi^{2}\right\rangle^{p+1 / 2}}=0 \text { and } \frac{\left\langle\psi^{2 p}\right\rangle}{\left\langle\psi^{2}\right\rangle^{p}}=(2 p-1) ! !
$$

(The corresponding curve is drawn on Fig. 4). On the contrary if $\psi^{2}$ takes only the value 0 and 1 with probability $(1-\gamma)$ and $\gamma$ respectively then

$$
\frac{\left\langle\psi^{2 p}\right\rangle}{\left\langle\psi^{2}\right\rangle^{p}}=\frac{1}{\gamma^{p-1}} \text {. }
$$

On figure 4 this law would correspond to a straight line. The growth of the computed moments are much slower than for a Gaussian process for small values of the order of the derivative $(i=0,1,2)$ while it is fairly close to the Gaussian law for $i=3,4$. For larger $i$, the moments of $\phi^{(i)}$ grow faster than the Gaussian law. This suggests non trivial probability distribution for each of the (local) quantities we have studied. The strong dependence with $i$ will be reconsidered in the next paragraph by investigating Fourier components of the field $\phi$. Finally let us mention the surprising distribution of the curves in figure 4 , which suggests a "pairing " of the moments

$$
\frac{\left\langle\phi^{(2 i+1)}(L / 2)^{2 p}\right\rangle}{\left\langle\phi^{(2 i+1)}(L / 2)^{2}\right\rangle^{p}} \text { and } \frac{\left\langle\phi^{(2 i)}(L / 2)^{2 p}\right\rangle}{\left\langle\phi^{(2 i)}(L / 2)^{2}\right\rangle^{p}} ;
$$

a property we could not explain.

\section{Multipoint statistics.}

Many theoretical approaches of fully developed turbulence rest on some assumptions, concerning the probability distribution of the Fourier components of the system. In particular, some simple probability distribution like Gaussian, or Log normal laws are often invoked. We report here some numerical results concerning the space Fourier transform of $\phi$ :

$$
\phi(k, t)=\frac{1}{2 \pi} \int_{0}^{L} \phi(x, t) \mathrm{e}^{i k x} \mathrm{~d} x .
$$

(In the subsection we have chosen periodic boundary conditions. However, it is also possible to define the Fourier transform of $\phi$ with boundary conditions :

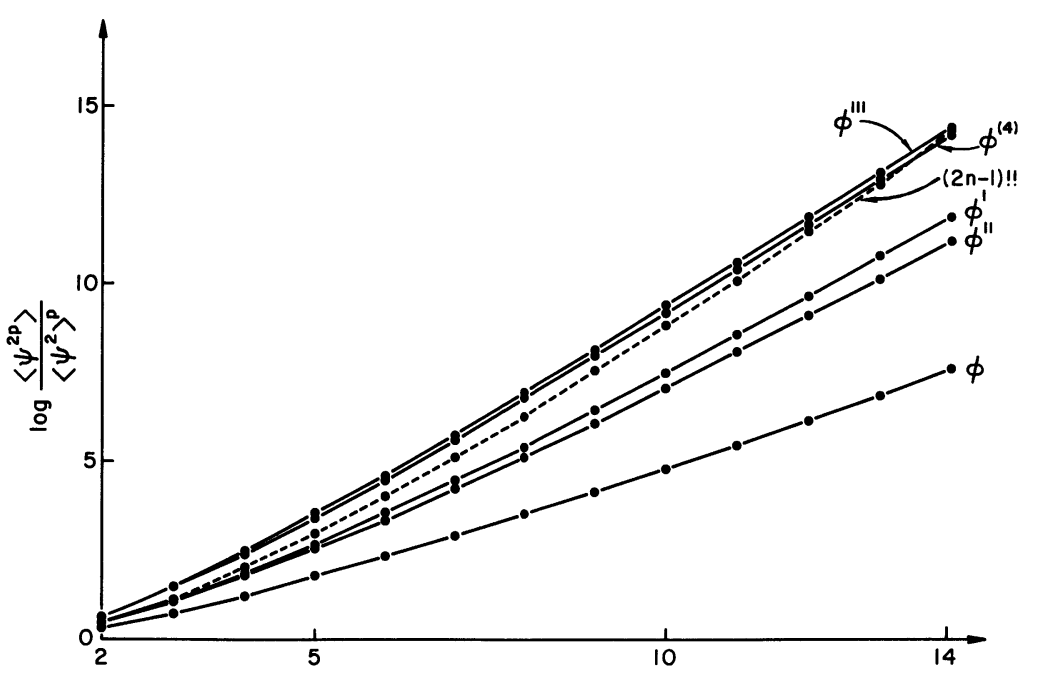

Fig. 4. - The logarithms of dimensionless ratios $\frac{\left\langle\psi^{2 n}\right\rangle}{\left\langle\psi^{2}\right\rangle^{n}}$ as a function of $n$ for several spatial derivatives of $\phi$ in the bulk regime. 
$\phi=\partial \phi / \partial x=0, x=0, L$; we have checked that for large values of $L$ the qualitative behaviour reported below is the same). In this section, the importance of the finite size of the system is stressed. The mean value of $|\phi(k)|^{2}$ has already been reported [8] and discussed [13]. According to the Wiener-Khinchin theorem, this mean value is nothing but the Fourier transform of the two point correlation function :

$$
\left\langle|\phi(k)|^{2}\right\rangle=\frac{1}{2 \pi L} \int_{0}^{L}\left\langle\phi\left(x_{1}, t\right) \phi\left(x_{2}, t\right)\right\rangle \mathrm{e}^{i k\left(x_{2}-x_{1}\right)} \mathrm{d} x_{1} \mathrm{~d} x_{2} .
$$

Similarly the higher moments of $\phi(k)$ provide information on the $n$ points correlation functions. We have considered $|\phi(k)|^{2 n}$ which satisfies :

$$
\left\langle|\phi(k)|^{2 n}\right\rangle=\frac{1}{(2 \pi L)^{n}} \int_{0}^{L}\left\langle\phi\left(x_{1}, t\right) \ldots \phi\left(x_{2 n}, t\right)\right\rangle \mathrm{e}^{i k}\left(\sum_{p=1}^{2 n}(-1)^{p} x_{p}\right) \mathrm{d} x_{1} \ldots \mathrm{d} x_{2 n} .
$$

If the factorization property $\left\langle\phi\left(x_{1}\right) \ldots \phi\left(x_{2 n}\right)\right\rangle=\sum^{\prime} \pi^{\prime}\left\langle\phi\left(x_{i}\right) \phi\left(x_{j}\right)\right\rangle$ holds one obtains $\left\langle|\phi(k)|^{2 n}\right\rangle=$ $\left\langle|\phi(k)|^{2}\right\rangle^{n}$ (where $\sum^{\prime}$ denotes the sum over all particions of $(1, \ldots, 2 n)$ into pairs, and $\pi^{\prime}$ denotes the product over these pairs).

This property suggests a Gaussian distribution :

$$
p\left(\phi\left(k_{1}\right) \ldots \phi\left(k_{n}\right)\right)=A \exp -\left(\frac{\left|\phi\left(k_{1}\right)\right|^{2}}{\left\langle\left|\phi\left(k_{1}\right)\right|^{2}\right\rangle}+\cdots+\frac{\left|\phi\left(k_{n}\right)\right|^{2}}{\left\langle\left|\phi\left(k_{n}\right)\right|^{2}\right\rangle}\right)
$$

(though the value of the moments of a distribution does not unequivocally determine a probability distribution). This distribution could be understood as a consequence of the central limit theorem. Indeed if one considers a sum of $N$ random complex terms : $S=\sum_{i=1}^{N} a_{i}$, with $\left\langle a_{i}\right\rangle=0$ and $\left\langle a_{i} a_{j}^{*}\right\rangle=f(|i-j|)$, the calculation of the $(2 n)$ th momentum of $S$ leads to a quantity which is in large $N$ limit of the order $n !\left\langle|S|^{2}\right\rangle^{n}$, as long as the other correlations $\left\langle a_{i_{1}} \ldots a_{i_{p}} \ldots a_{i_{p+1}}^{*} \ldots a_{i_{2_{p}}}^{*}\right\rangle$ are factorized. So the quantities $\frac{\left\langle|\phi(k)|^{2 n}\right\rangle}{n !\left\langle|\phi(k)|^{2}\right\rangle^{n}}$ measure a lack of factorization of the correlation functions, or equivalently the non Gaussian character of the probability distribution. Let us also mention that the non Gaussian character of the fluctuations in dynamical systems has already been discussed by Ruelle [20]. The existence of strange attractors, as suggested by numerical experiments, makes normal distributions very unlikely. With periodic boundary conditions it is possible to write (formally) the K.S.E. as an infinite dimensional dynamical system :

$$
\frac{\mathrm{d}(\phi(k))}{\mathrm{d} t}=+\left(k^{2}-k^{4}\right) \phi(k)+\sum_{k^{1}+k^{11}=k} i k^{1} \phi\left(k^{1}\right) \phi\left(k^{11}\right) .
$$

From these considerations it would not be surprising that the distribution of $\phi(k)$ be non Gaussian.

Our numerical results were obtained from a sample of 20,000 spectra separated by the time interval $\Delta t=0.4$ and corresponding to a size $L=128$. The value of the spatial mesh size was $\Delta x=0.25$; this allows a reasonable resolution up to $k=4$. Some typical curves are drawn on figure 5. From these results it can be deduced that the distribution of $|\phi(k)|^{2}$ is non Gaussian, the discrepancy increasing with $k$, for large values of $k$. Moreover our numerical results suggest the following empirical relation :

$$
\frac{\left\langle|\phi(k)|^{2 p}\right\rangle}{p !\left\langle|\phi(k)|^{2}\right\rangle^{p}}=\gamma(k)^{2 p-3}
$$

which is fairly well satisfied up to $p=5$.

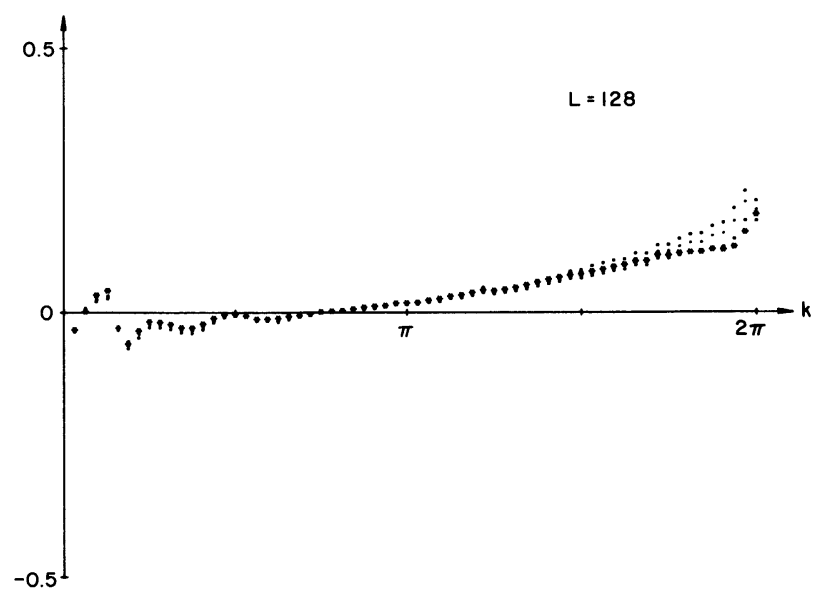

Fig. 5. - The logarithms of the dimensionless ratios $\left(\frac{\left\langle|\phi(k)|^{2 n}\right\rangle^{1 / 2 n-1}}{n !\left\langle|\phi(k)|^{2}\right\rangle^{n}}\right)$ as a function of $k$ for $n=2,3,4,5$. The 4 curves are almost perfectly superposable. At high wavenumbers, these ratios grow monotonously. 
A possible explanation of these phenomena involves the theory proposed by Frisch and Morf [21]. It can be seen that the growth of the dimensionless ratios $\left\langle|\phi(k)|^{2 n}\right\rangle \mid\left\langle|\phi(k)|^{2}\right\rangle^{n}$ occurs for $k$ large enough, in a region where $\left\langle|\phi(k)|^{2}\right\rangle$ decreases exponentially (" dissipative range "). The existence of singularities in the complex extension of the $x$ axis for the solution of the K.S.E., located at a distance $\delta$ from the real axis gives rise to a high wavenumber part of the Fourier spectrum decreasing exponentially with $k=S(k) \sim \mathrm{e}^{-k \delta}$ (up to algebraic terms). The probably irregular distribution of the singularities induces fluctuations of $|\phi(k)|^{2}$ which are increasingly intermittent at high $k$ (see the discussion of Kraichnan [22]). In fact the knowledge of the functions $\frac{\left\langle\left|\phi_{k}\right|^{2 n}\right\rangle}{\left\langle\left|\phi_{k}\right|^{2}\right\rangle^{n}}$ provides some information on the distribution of singularities in the complex plane. Unfortunately even for simple dynamical systems (as for example the Lorenz equations) nothing is known about the probability distribution of these singularities in the complex plane. Let us mention that the components of the Fourier spectrum do not provide the most efficient way to investigate small scale intermittency effects. As explained in reference [21] it is more appropriate to use a broad pass (or high pass) filter; the signal becomes more and more intermittent when the characteristic frequency is increased. Similar tests have been done by Manneville [9] to study temporal small scale intermittency in the Kuramoto-Sivashinsky equations, or by Gagne [23] in real fluids. The point of view developed in this paper is slightly different. Other calculations could confirm unambiguously the existence of small scale intermittency effects, however we argue that the high wavenumber behaviour of the $|\phi(k)|^{n}$ has to do with the same kind of phenomenon. Another explanation of the facts observed involves an evaluation of $\left\langle\left|\phi_{k}\right|^{2 n}\right\rangle$ in terms of the space correlation functions. If one writes :

then

$$
\left\langle\phi\left(x_{1}\right) \ldots \phi\left(x_{m}\right)\right\rangle=\sum^{\prime} \pi^{\prime}\left\langle\phi\left(x_{i}\right) \phi\left(x_{j}\right)\right\rangle+G\left(x_{1} \ldots x_{m}\right)
$$

$$
\begin{aligned}
\int \mathrm{d} x_{1} \ldots \mathrm{d} x_{2 n}\left\langle\phi\left(x_{1}\right) \ldots \phi\left(x_{2 n}\right)\right\rangle \mathrm{e}^{i k\left(x_{1}+\cdots+x_{n}-\left(x_{n+1}+\cdots+x_{2 n}\right)\right)}=n !\left(\int_{0}^{L} \mathrm{~d} x_{i} \mathrm{~d} x_{j}\left\langle\phi\left(x_{i}\right) \phi\left(x_{j}\right)\right\rangle \mathrm{e}^{i k\left(x_{i}-x_{j}\right)}\right)^{n}+ \\
+\int \mathrm{d} x_{1} \ldots \mathrm{d} x_{2 n} G\left(x_{1} \ldots x_{2 n}\right) \mathrm{e}^{i k\left(x_{1}+\cdots+x_{n}-\left(x_{n+1}+\cdots+x_{2 n}\right)\right)}=n ! L^{n}\left\langle|\phi k|^{2}\right\rangle^{n}+I_{n}(k) .
\end{aligned}
$$

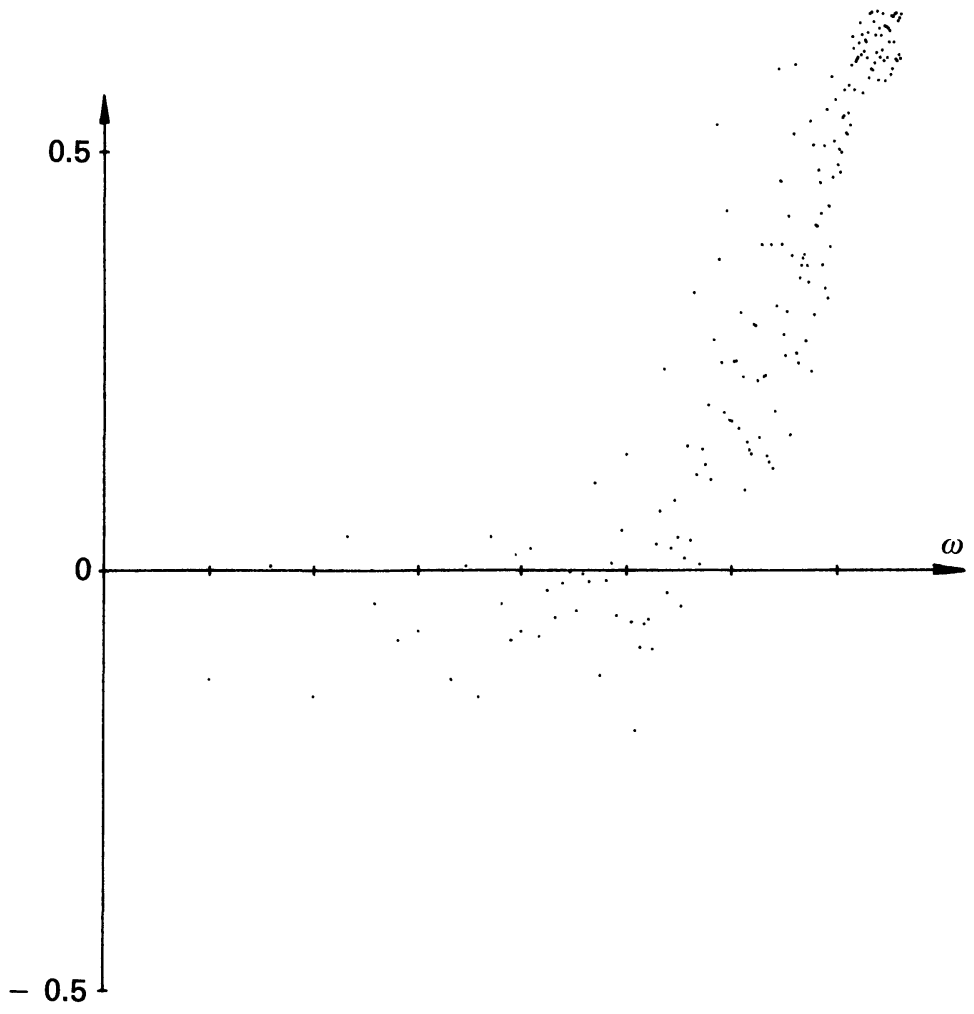

Fig. 6. - The logarithm of the dimensionless ratio $\left(\frac{\left\langle\phi \omega^{8}\right\rangle}{\phi !\left\langle\phi \omega^{2}\right\rangle^{4}}\right)$ as a function of $\omega$. For small frequencies the distribution of $\phi \omega$ are almost Gaussian while at larger frequencies the distribution is more intermittent. 
For large $k\left\langle\left|\phi_{k}\right|^{2}\right\rangle$ decays exponentially (this can be easily understood, since the Fourier components of a $C^{\infty}$ periodic function decrease faster than any power of $k$, more rigorous arguments can be found in Frisch and Morf [21]). On the other hand the shape of the solution (Fig. 1) suggests there is no long range order in the system; so that $G\left(x_{1} \ldots x_{2 n}\right)$ is negligible unless at least three $x_{i}$ are close to each other. Thus $I_{n}(k)$ is of order $L^{n-1}$ (or less). For small $k\left\langle\left|\phi_{k}\right|^{2}\right\rangle$ is large enough so that

$$
\left\langle\left|\phi_{k}\right|^{2 n}\right\rangle \approx n !\left\langle\left|\phi_{k}\right|^{2}\right\rangle^{n} \text {. }
$$

On the contrary for larger wavenumbers, $\left\langle\left|\phi_{k}\right|^{2}\right\rangle$ is very small. Due to non trivial correlations at short distances, $I_{n}(k)$ can be significant even for large $k$. Thus $\left\langle\left|\phi_{k}\right|^{2 n}\right\rangle \gg n !\left\langle\left|\phi_{k}\right|^{2}\right\rangle^{n}$ for large enough $k$.

This argument involves the size $L$ of the system. In particular for very large values of $L$ one expects that the deviations from Gaussian distribution are very small for finite values of $k$. This effect was indeed observed.

In conclusion, we have verified the non Gaussian character of the fluctuations of the dynamic variables $\phi(k)$ for finite systems which arises from quite general considerations. In particular for large wavenumbers this property results from a lack of factorization of the correlation functions due to the deterministic nature of the equations. In the large $L$ limit, the Gaussian distribution is recovered. We now turn to multitime correlation functions.

\section{Time correlation statistics and spectral analysis.}

For a random signal $s(t)$ it is also possible to use spectral analysis (see, e.g. Rice [24]). Given a time interval $[0, T]$, one writes :

$$
s(t)=\frac{x_{0}}{2}+\sum_{n=1}^{\infty}\left(x_{n} \cos \frac{2 \pi n t}{T}+y_{n} \sin \frac{2 \pi n t}{T}\right) .
$$

Where the $x_{i}$ and $y_{j}$ are random quantities. The main interest of this study is to gain insight on the time correlation functions of the signal. By definition, the auto-correlation function of $s($.$) is :$

$H(t)=\lim _{T \rightarrow \infty} \frac{1}{T} \int_{t_{0}}^{t_{0}+T} \mathrm{~d} t^{1}\left(s\left(t^{1}\right)-\langle s\rangle\right)\left(s\left(t^{1}+t\right)-\langle s\rangle\right)$

$\langle s\rangle$ being the average value of $s($.$) . The Wiener-$ Khinchin theorem asserts that :

$$
\begin{gathered}
\lim _{T \rightarrow \infty} \frac{1}{T}\left|\widetilde{S}_{T}(\omega)\right|^{2}=\int_{-\infty}^{+\infty} \mathrm{e}^{i \omega t} H(t) \mathrm{d} t \\
\widetilde{S}_{T}(\omega)=\int_{t_{0}}^{t_{0}+T} \mathrm{~d} z^{1} \mathrm{e}^{i \omega \tau^{1}} s\left(\tau^{1}\right) .
\end{gathered}
$$

Consequently in the large $T$ limit, the mean values of $\left(x_{i}^{2}+y_{i}^{2}\right)$ are nothing but the Fourier components of the auto correlation functions. Similarly for any integer $n$, the quantity $\left(\left|x_{i}\right|^{2}+\left|y_{i}\right|^{2}\right)^{n}$ is related to the $2 n$ points correlation function of the signal; in particular, the property of factorization of the $2 n$ times correlation functions implies that

$$
\frac{\left\langle\left(\left|x_{i}\right|^{2}+\left|y_{i}\right|^{2}\right)^{n}\right\rangle}{\left\langle\left(\left|x_{i}\right|^{2}+\left|y_{i}\right|^{2}\right)\right\rangle^{n}}=n !
$$

(see the arguments of the previous section). Some results concerning the two times correlation function of fluctuating quantities governed by the K.S.E. have already been obtained by Manneville [9]. In this section we are dealing with $2 n$ times correlation functions of a local quantity : $s(t)=\phi\left(x_{0}, t\right)\left(x_{0}\right.$ being a given point, chosen far away from the boundaries, at which $\phi=\partial \phi / \partial x=0$ ). The results presented below have been obtained as the accumulation of 100 individual fast Fourier transforms of consecutive series of $M=256$ samples taken every $\Delta t=0.8$. These data were multiplied by a cosine bell in order to avoid spurious effects due to the non periodicity of the signal. So the time interval was $T=M \Delta T=$ 204.8 , which is very large compared to the characteristic times in the system (of the order of the inverse of the maximum linear growth rate $\tau \sim 4$ ). Our statistics are less accurate than in the previous part since a time spectrum requires many elementary integrations of the K.S.E. The results are presented in figure 6. The low frequency part of the spectrum is almost governed by a Gaussian statistics. This can be checked from the computed values of $\frac{\left\langle|\phi \omega|^{2 p}\right\rangle}{\left\langle|\phi \omega|^{2}\right\rangle^{p}}$ and also from the histograms we have plotted (see Fig. 7). For $\omega$ large enough deviations from Gaussian distribution occur. These results rather similar to the

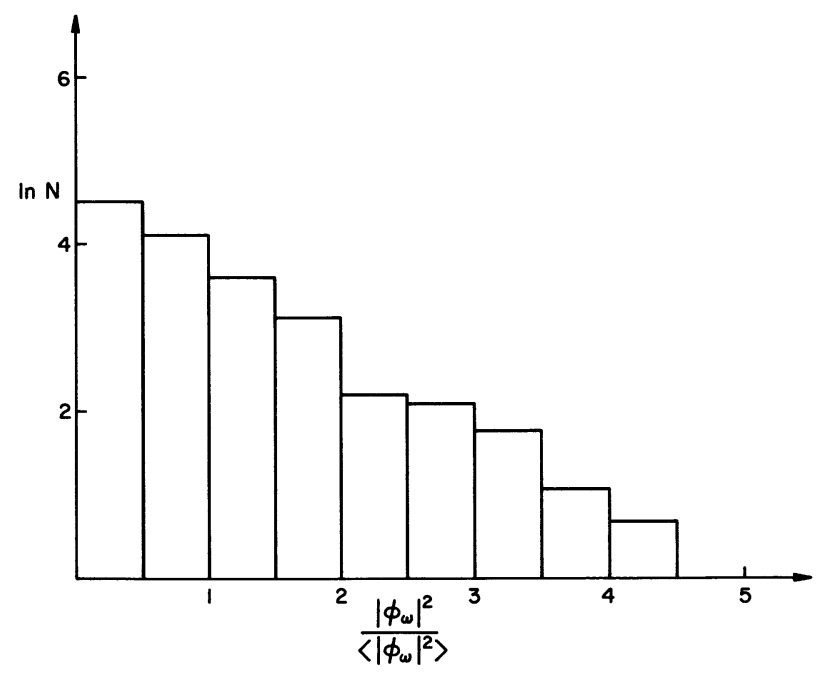

Fig. 7. - Histogram representing the distribution of $\frac{|\phi \omega|^{2}}{\left\langle|\phi \omega|^{2}\right\rangle}$ for $\omega \sim 0.03$. The distribution of $|\phi \omega|^{2}$ is very close to a Gaussian distribution. 
previous one deserve the same comments. In particular, due to the deterministic nature of the system, the $2 p$-times correlation function $\left\langle s\left(t_{1}\right) \ldots s\left(t_{2 p}\right)\right\rangle$ of the system are very likely to be quite different from the factorized form : $\sum^{\prime} \pi^{\prime}\left\langle s\left(t_{i}\right) s\left(t_{j}\right)\right\rangle$ as soon as two of the $t_{i}$ are close enough. The same reasoning as before explains why for large frequencies, the dimensionless ratios $\frac{\left\langle\left|\phi_{-}\right|^{2 n}\right\rangle}{\left\langle|\phi \omega|^{2}\right\rangle^{n}}$ are much larger than $n$ !

We have also tested these ideas on another deterministic model which displays a chaotic behaviour; the Lorenz model (Lorenz [25])

$$
\dot{x}=\sigma(x-y) \quad \dot{y}=-x y+r x-y \quad \dot{z}=x y-b z .
$$

With $\sigma=10, b=8 / 3$ and $r=28$. For numerical calculations a 4th order Runge Kutta algorithm has been used. The time step was $\Delta t=0.005$ which is very small compared to the characteristic time scale of the system, $\tau \sim 1$. The spectra have been computed from a set of $N$ points separated by $\Delta t=0.02(N=2048$ or 4096 , corresponding to a total time $T=40.96$ or $T=81.92$ ). We present results concerning the-fluctuations of the $x$ variable, similar results were obtained for $y$ or $z$. The mean value of $|\phi \omega|^{2}$ is plotted in figure 8 , while the values of the dimensionless ratios $\frac{\left\langle|\phi \omega|^{2 p}\right\rangle}{p !\left\langle|\phi \omega|^{2}\right\rangle^{p}}$ are presented on figure 9 for $N=2048$, and on figure 10 for $N=4096$. The low frequency part of the spectra is almost governed by a Gaussian statistics. For larger $\omega$, slight deviations from Gaussian distribution appear. The same empirical relation as in the previous paragraph holds :

$$
\left.\frac{\left\langle|\phi \omega|^{2 p}\right\rangle}{\left\langle|\phi \omega|^{2}\right\rangle^{p}}=p ! \gamma(\omega)^{2 p-3} \quad \text { (for } p>2\right) \text {. }
$$

The difference in the shapes of figure 9 and 10 can be explained as follows. If one assumes that the high

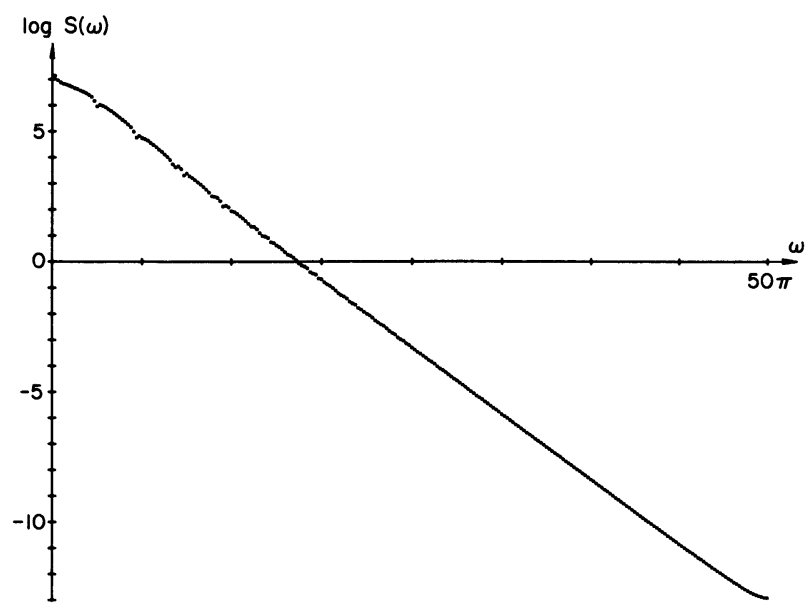

Fig. 8. - The power spectrum for the variable $x$ in the Lorenz system.
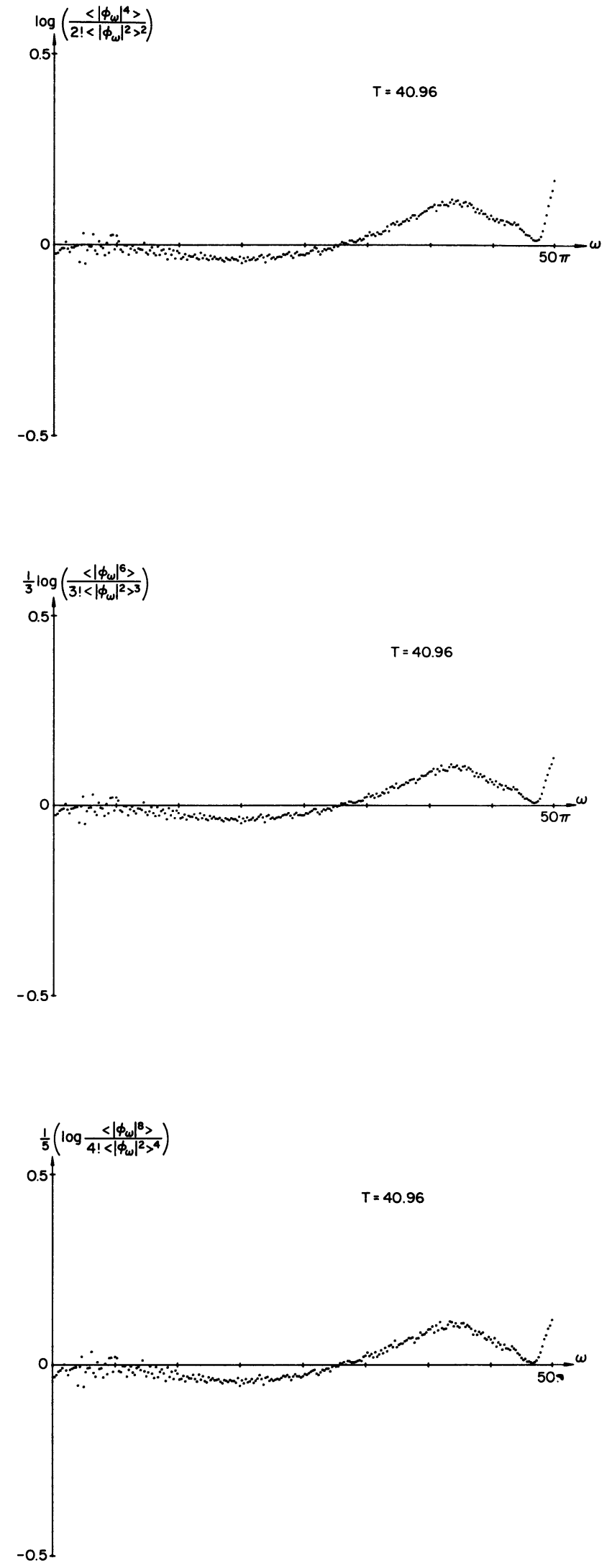

Fig. 9. - The logarithm of the dimensionless ratios $\frac{\left\langle|x \omega|^{2 n}\right\rangle}{\left\langle|x \omega|^{2}\right\rangle^{n}}$ as a function of $\omega, n=2,3,4$. The time sampling is $T=40.96$. (The high frequency behaviour is due to spurious effects of basic non periodicity of the signal.) 

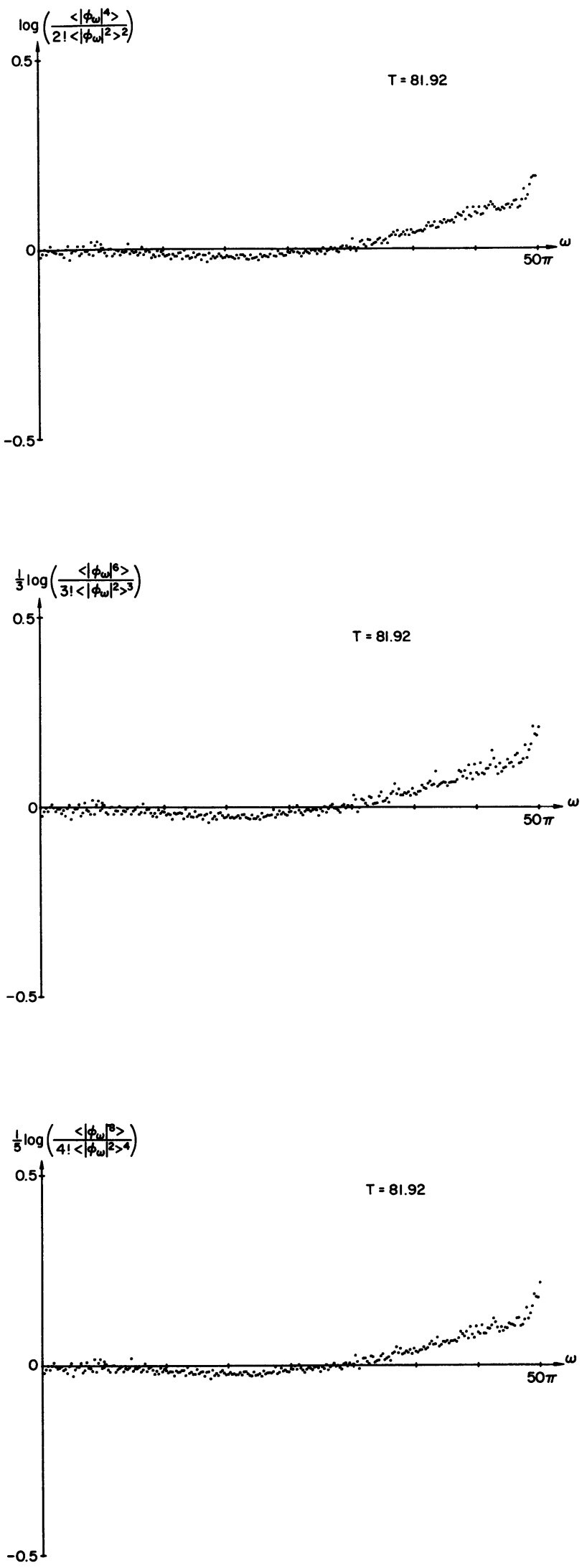

Fig. 10. - The logarithms of the dimensionless ratios $\frac{\left\langle|x \omega|^{2 n}\right\rangle}{\left\langle|x \omega|^{2}\right\rangle^{n}}$ as a function of $\omega, n=2,3,4$. The time sampling is $T=81.92$. frequency growth of the moments is induced by the closest singularities to the real axis for a given value of $T$ one is more sensitive to the occurrence of scarce singularities than for the value $T / 2$. Otherwise an evaluation of the Fourier transform of the $2 n$ points correlation function, similar to the estimates of the last paragraph, explicitly depends on the time sampling $T$.

This study exhibits similar behaviour for several deterministic equations. In these systems the low frequency fluctuations are governed by an almost Gaussian probability law while the high frequency fluctuations are no longer Gaussian. The latter property is related to the non trivial small time correlations induced by the deterministic nature of the system; while the former has to do with large time factorization of the correlation functions. Let us also mention that similar results have been obtained in the case of Langevin's equation (see Chandrasekhar [26]). For the Brownian motion, the well known laws of diffusion are asymptotically valid; the decay of the correlations, induced by the "deterministic " nature of the Langevin's equation (albeit through a random term) requires a while. It would be interesting to check these properties on real, experimental systems, though it could be fairly difficult to investigate.

\section{Correlation functions testing the time symmetry of the fluctuations.}

We have suggested in the previous paragraph that the deterministic nature of the equations of motion could be responsible for the observed facts. We will try to make this point more precise by using correlation functions testing the time reversal symmetry ( $t$-symmetry) of a signal (see [18] for a detailed introduction). If one considers a stationary random process described by a real fluctuating quantity $s(t)$, some are sensitive to it (e.g. $\langle s(t+\tau) s(t) s(t-2 \tau)\rangle)$. Such correlation functions can provide further details on ill-understood dynamical processes occurring in systems with many degrees of freedom, such as equations describing hydrodynamic turbulence. The spatial power spectrum of the K.S.E. have been discussed in terms of simple dynamical models, involving local energy transfer in wavenumber space [13]. We have thus investigated the $t$-symmetry of the fluctuations in the Fourier space. Let us define :

$$
\begin{aligned}
& \psi_{k}(\tau)= \\
& =\frac{\left\langle\left|\phi_{k}\right|^{4}(\tau)\left|\phi_{k}\right|^{2}(t+\tau)-\left|\phi_{k}\right|^{4}(t+\tau)\left|\phi_{k}\right|^{2}(t)\right\rangle}{\left\langle\left|\phi_{k}(t)\right|^{2}\right\rangle^{3}} .
\end{aligned}
$$

The function $\psi$ is a function of both time and wavenumber. Our results have been obtained by integrating the K.S.E., starting from one initial condition and from 20,000 values of the solutions separated by $\Delta t=0.5$; they are presented on figure 11 . 

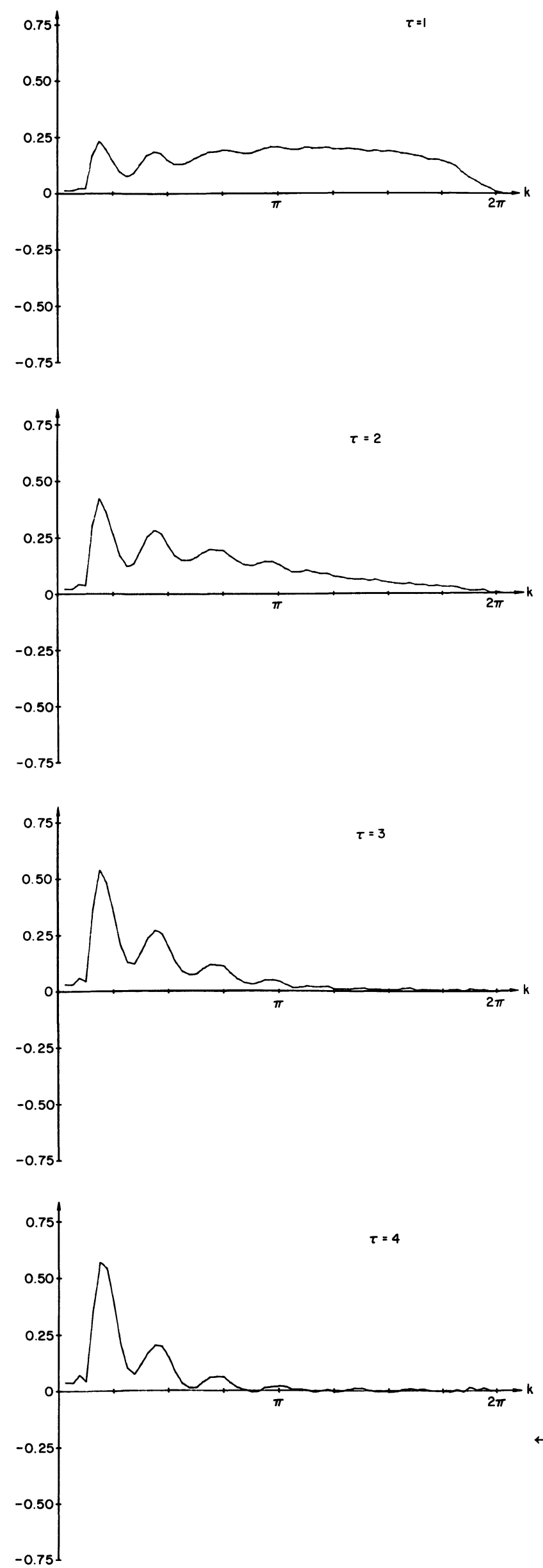

(Similar results have been obtained for different powers of $\left.\left|\phi_{k}\right|\right)$. For $\tau$ small enough $(\tau \sim 1,2)$ the large wavenumber fluctuations $(k \gg 1)$ display an obvious lack of $t$-symmetry. This property disappears for larger $\tau$, and $\psi_{k}(\tau) \rightarrow 0$ for all wavenumbers we have considered. For a given value of $\tau, \psi_{k}(\tau)$ is a function of $k$ which exhibits small oscillations. These results suggest that irreversible effects occur mainly in the "dissipation range ", which is consistent with the intuitively irreversible character of dissipative processes.

The previously mentioned property of factorization of time correlation functions explains why $\lim \psi_{k}(\tau)=0$, for all $k$. It is more difficult to explain the oscillation of $\psi_{k}(\tau)$ with $k$, or even the sign of $\psi_{k}(\tau)$ at large $k$.

The lack of $t$-symmetry for large wavenumbers can be related to the non Gaussian character of the fluctuations. Loosely speaking, the dynamical process (dissipation) induces strong constraints on $\phi$ for $k>1$ which induces a non Gaussian distribution of the fluctuations. In order to identify a possible transfer phenomenon in wavenumber space we have also considered a correlation function which should test the sense of flow of energy in wavenumber space [8] :

$$
\begin{aligned}
& \chi_{k, k+\Delta k}(\tau)= \\
& \quad=\frac{\left\langle|\phi(k, t)|^{2}|\phi(k+\Delta k, t+\tau)|^{2}-|\phi(k+\Delta k, t)|^{2}|\phi(k, t+\tau)|^{2}\right\rangle}{\left\langle|\phi(k, t)|^{2}\right\rangle\left\langle|\phi(k+\Delta k, t)|^{2}\right\rangle} .
\end{aligned}
$$

One of the main interest of such a correlation function is to detect a possible transfer of energy. Imagine that energy flows from low wavenumbers to high wavenumbers, with a constant velocity, $c=\mathrm{d} k / \mathrm{d} t$. This flow will induce large correlations between the value of the energy at wavenumber $k$, at time $t$ and the value of the energy at a larger wavenumber $k+\Delta k$, and later times, $t+\Delta t\left(\Delta t \sim \frac{\Delta k}{c}\right)$. On the contrary the correlation between the values of the energy at wavenumber $k+\Delta k$, at time $t$ and its value at wavenumber $k, t+\frac{\Delta k}{c}$ will be much weaker. Thus it is expected that a flow of energy to high wavenumbers induces positive values of $\chi_{k, k+\Delta k}(\tau)$ for some values of $\tau>0$. Let us also mention that it is possible to investigate the energy transfer by computing the three points correlation function which occurs in the energy budget equation, as has been done for the Navier-Stokes equations by Siggia [27].

$\leftarrow$ Fig. 11. - The correlation function

$$
\psi_{k}(\tau)=\frac{\left\langle\left|\phi_{k}(t)\right|^{4}\left|\phi_{k}(t+\tau)\right|^{2}-\left|\phi_{k}(t+\tau)\right|^{4}\left|\phi_{k}(t)\right|^{2}\right\rangle}{\left\langle\left|\phi_{k}(t)\right|^{2}\right\rangle^{3}}
$$

for $\tau=1,2,3$ and 4 . 

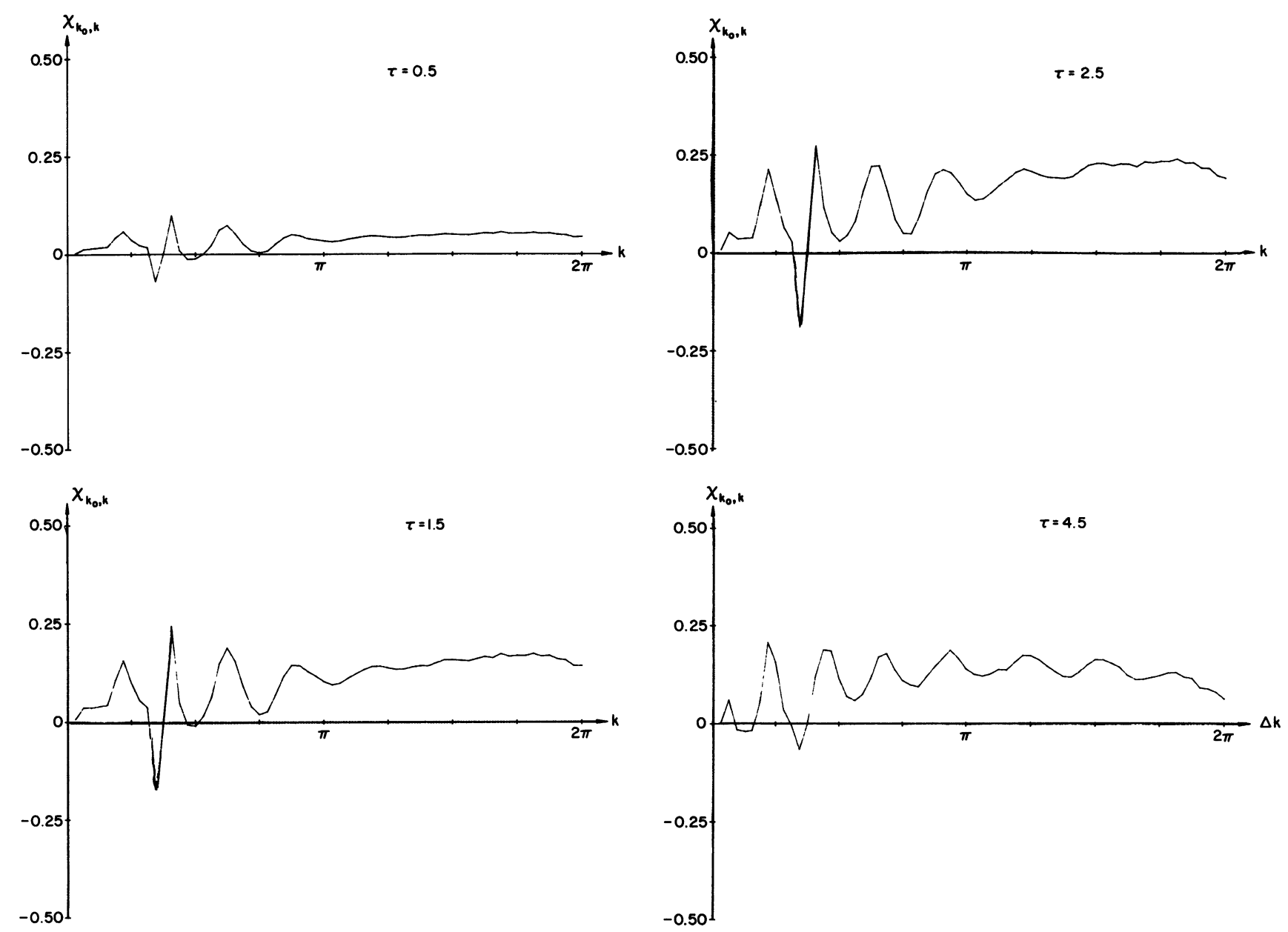

Fig. 12. - The correlation function $\chi_{k_{0, k}}(\tau)$ as a function of $k$ for $k_{0}=1.1$ and $\tau=0.5,1.5,2.5$ and 4.5.

On figures 12 are plotted $\chi_{k_{0}, k}(\tau)$ as a function of $k$ for different values of $\tau, k_{0}$ being chosen in the algebraic part of the spectrum. The shape of one of these curves, which displays large oscillations for small enough $k$, is roughly unchanged through the whole evolution. For $\Delta k$ large enough (corresponding to a transfer of spectrum through the dissipative zone) $\chi_{k, k+\Delta k}$ no longer presents oscillations, and takes large enough positive values. The former fact is not surprising since a transfer of energy ot the dissipative domain must occur according to very simple arguments. The similarity of the shapes of the curves of figure 12 provides also some insight on the mechanism of energy transfer. If one imagines that energy is transferred by a local process in wavenumber space, then one should observe a delay before energy reaches the very high wavenumbers. As the characteristic time in the system is of order 1 , such an effect should be observable in our figures. On the contrary our numerical experiments suggest that the transfer of energy occurs in a different way. The existence of localized structures and their relevance to the dynamical process has already been discussed from a different standpoint [13]. The results we present in this paper corroborate the existence of a transfer of energy involving localized structure. In fact the similarity of the different curves of figure 12 suggest that all the wavenumbers in Fourier space receive energy simultaneously. This can be understood if one assumes the existence of basic structures in the system, deeply related to the dynamical processes. In particular the transfer of energy through high wavenumbers in the spectrum even after a short lapse of time should be reminiscent of the small scale structure of these hypothetical structures. Actually our argument is not a formal proof of the existence of these structures, it is however very suggestive.

It would be worth to do similar calculations in more realistic models of turbulence. Our results suggest that this could shed some light on the still ill understood mechanisms of energy transfer.

\section{Concluding remarks.}

First we would like to stress some of our results. The facts observed concerning the large wavenumber and high frequency fluctuations are a manifestation of small scale intermittency. We have indeed observed 
similar properties for quite different phenomena. The explanations we have proposed.allow us to relate these properties to the lack of factorization of the correlation functions at short distances, a very reasonable property. In the case of the K.S.E. it has also been shown that the large wavenumber fluctuations are almost Gaussian. We have strong evidence that this property is true in the infinite size limit $(L \rightarrow \infty)$. The low frequency part of the temporal spectrum shares these properties. Other results concerning correlation functions testing the time-symmetry of the fluctuations have also been obtained. Our study corroborates the idea of a transfer of energy non local in wavenumber space which would involve localized structures.

Beyond these preliminary results concerning the K.S.E. many interesting questions remain open. Up to now a few incomplete theories have been proposed to explain the statistical features of systems with many degrees of freedom, such as hydrodynamic turbulence. For example the approaches in term of fractal set [28] have a poor dynamical support. Since Kolmogorov's approaches, or more recent works on inertial subrange the probability distribution of the fluctuations is of great interest. Though the connection between the K.S.E. and the Navier-Stokes equations is quite loose, our study provides accurate enough results which should provide a basis for further theoretical investigations. We also believe that the investigation of correlation functions testing the time symmetry of the fluctuations should improve our current understanding of systems displaying temporal and spatial chaos.

\section{Acknowledgments.}

I am very indebted to Y. Pomeau for his suggestions, encouraging remarks and for his constant interest in this work. I have benefited from many discussions with P. Manneville especially about the redaction of the present paper. I wish also to thank E. D. Siggia for a careful reading of the manuscript. A grant of computer time from Service de Physique Théorique, Saclay, France, where the main part of this work has been done, and from the CCVR, Palaiseau, France is gratefully acknowledged.

\section{References}

[1] Deissler, R. G., Rev. Mod. Phys. 56 (1984) 223.

[2] Anselmet, F., Gagne, Y., Hopfinger, E. J. and Antonia, R. A., J. Fluid Mech. 140 (1984) 63.

[3] Kuramoto, Y., Suppl. Prog. Theor. Phys. 64 (1978) 346.

[4] Sivashinsky, G. I., Acta Astron. 4 (1977) 1177.

[5] Sivashinsky, G. I., Acta Astron. 6 (1979) 569.

[6] Sivashinsky, G. I. and Michelson, D. M., Prog. Teor. Physics 63 (1980) 2112.

[7] Shlang, T. and Sivashinsky G. I., J. Physique 43 (1982) 659.

[8] Yamada, T. and Kuramoto, Y., Prog. Theor. Phys. 56 (1976) 681

[9] Manneville, P., Phys. Lett. 84A (1981) 129.

[10] YAKHOt, V., Phys. Rev. A 24 (1981) 642.

[11] Fujisaka, H. and Yamada, T., Prog. Theor. Phys. 57 (1977) 734

[12] Nicolaenko, B., Scheurer, B. and Temam, R., C.R. Hebd. Séan. Acad. Sci. Série I 298 (1984) 23.

[13] Pomeau, Y., Pumir, A. and Pelcé, P., J. Stat. Phys. (1984) (to be published).

[14] Ruelle, D., Ann. N. Y. Acad. Sci. 357 (1980) 1.

[15] I am indebted to one of my referees for this remark.
For further details concerning this point, see the lecture presented at the "Enrico Fermi " summer school, 1983 (Varenna, Italy) by U. Frisch (to published by North Holland).

[16] Monin, A. S. and Yaglom, A. M., Statistical Fluid Dynamics, Massachusetts Institute of Technology Press (1975).

[17] TAYlor, G. I., Proc. Lond. Math. Soc. 20 (1921) 196.

[18] Pomeau, Y., J. Physique 43 (1982) 859.

[19] Batchelor, G. K. and Townsend, A. A., Proc. R. Soc. A 199 (1949) 238.

[20] Ruelle, D., Prog. Theor. Phys. Suppl. 64 (1978) 339.

[21] FrisCh, U. and Morf, R., Phys. Rev. A 23 (1981) 2673.

[22] Kraichnan, R. H., Phys. Fluids 10 (1967) 2080.

[23] GAGNE, Y., thèse de docteur ingénieur (1980) (unpublished).

[24] RICE, S. O., in Selected papers on noise and stochastic processes, N. Wax, editor (Dover, New York) 1954.

[25] LorenZ, E. N., J. Atmos. Sci. 20 (1963) 130.

[26] Chandrasekhar, S., Rev. Mod. Phys. 15 (1943) 1.

[27] Siggia, E. D., J. Fluid Mech. 107 (1981) 375.

[28] Mandelbrot, B., J. Fluid Mech. 62 (1974) 331. 